\title{
TYPICALITY IN PURE WAVE MECHANICS
}

\author{
JEFFREY A. BARRETT
}

\begin{abstract}
Hugh Everett III's pure wave mechanics is a deterministic physical theory with no probabilities. He nevertheless sought to show how his theory might be understood as making the same statistical predictions as the standard collapse formulation of quantum mechanics. We will consider Everett's argument for pure wave mechanics, how it depends on the notion of branch typicality, and the relationship between the predictions of pure wave mechanics and the standard quantum probabilities.
\end{abstract}

\section{INTRODUCTION}

Hugh Everett III $(1956 ; 1957)$ presented pure wave mechanics, sometimes referred to as the many-worlds interpretation, as a preferable alternative to both the Copenhagen and standard von Neumann-Dirac collapse formulations of quantum mechanics. While pure wave mechanics is a deterministic physical theory with no probabilities, Everett sought to show how the theory might be understood as making the standard quantum statistical predictions as appearances to observers who were themselves described by the theory. The key step in his argument was to show that there was a sense in which almost all quantum-mechanical branches of the total state will describe sequences of measurement results that are compatible with the standard statistical predictions of quantum mechanics. We will consider his argument, how it depends on the notion of branch typicality, and the relationship between the predictions of pure wave mechanics and the standard quantum probabilities.

Everett began his presentation of pure wave mechanics with a discussion of the difficulties that John von Neumann's $(1932 ; 1955)$ formulation of P. A. M. Dirac's (1930) description of quantum mechanics encounters. While the standard von Neumann-Dirac collapse formulation of quantum mechanics correctly predicts a broad range of counterintuitive phenomena and does so with extraordinary precision, Everett believed that it was logically inconsistent in the context of nested measurements. To illustrate the problem, he used the standard collapse theory to tell his version of what later became known as the Wigner's Friend story. He then characterized pure wave mechanics as a logically consistent simplification of the standard theory.

Date: January 31, 2016. 
Following von Neumann, Everett took the standard collapse formulation of quantum mechanics to be characterized by the following principles:

1. Representation of States: The state of a physical system $S$ is represented by a vector $\psi_{S}$ of unit length in a Hilbert space $\mathcal{H}$.

2. Representation of Observables: A physical observable $O$ is represented by a set of orthogonal vectors $\mathcal{O}$. These vectors represent the eigenstates of the observable, each corresponding to a different value.

3. Interpretation of States: A system $S$ has a determinate value for observable $O$ if and only if $\psi_{S} \in \mathcal{O}$.

\section{Dynamical Laws:}

I. Linear dynamics: If no measurement is made, the system $S$ evolves in a deterministic linear way: $\psi\left(t_{1}\right)_{S}=\hat{U}\left(t_{0}, t_{1}\right) \psi\left(t_{0}\right)_{S}$.

II. Nonlinear collapse dynamics: If a measurement is made, the system $S$ randomly, instantaneously, and nonlinearly jumps to an eigenstate of the observable being measured: the probability of jumping to $\phi_{S}$ when $O$ is measured is $|\psi \phi|^{2}$.

A salient feature of the theory is the fact that it has two dynamical laws. Both are standardly taken to be necessary to explain determinate measurement records. The deterministic linear dynamics (4I) explains quantum interference effects. The collapse dynamics (4II) explains the standard quantum statistics. It also explains how to update probabilities on the basis measurement results. Suppose one measures the $x$-spin of a spin- $1 / 2$ system $S$ that is initially in a eigenstate of $z$-spin and gets the result $x$-spin up. While the initial probability of $x$-spin up was $1 / 2$, if one repeats the $x$-spin measurement of $S$ without disturbing it, one will get an $x$-spin up result with probability 1 . The standard explanation is that $S$ is no longer in a $z$-spin eigenstate, but rather, is now in the $x$-spin up eigenstate as a result of the collapse dynamics (4II) describing the evolution of $S$ over the first measurement.

Everett examined what he called the question of the consistency of the standard theory in the context of an "amusing, but extremely hypothetical drama" (1956, 745). ${ }^{1}$ This was the original version of what has come to be known as the Wigner's Friend story. ${ }^{2}$ Everett used the story to argue that there is a contradiction between the two dynamical laws, and, hence that the standard formation of quantum mechanics is logically inconsistent.

\footnotetext{
${ }^{1}$ Everett first consider this story in a short working paper (1955b) that he wrote for his advisor John Wheeler.

${ }^{2}$ Eugene Wigner (1961) famously retold essentially the same story some years later in support of his own formulation of quantum mechanics, one where a collapse of the quantum-mechanical state occurs when a nonphysical mind apprehends the system. Given what he says about other formulations of quantum mechanics, there is good to suppose that Everett would have rejected Wigner's later reformulation of the standard collapse theory as ad hoc, nonphysical, and unnecessarily complex when compared with pure wave mechanics.
} 
Using Eugene Wigner's (1961) terminology, Everett described a friend $F$ in a state $|" r "\rangle_{F}$ ready to observe his measuring device and his measuring device $M$ in a state $\mid$ "r" $\rangle_{M}$ ready to measure $x$-spin of a spin- $1 / 2$ system $S$ that, in turn, begins in the state

$$
\alpha\left|\uparrow_{x}\right\rangle_{S}+\beta\left|\downarrow_{x}\right\rangle_{S} .
$$

Assuming that the friend $F$ is a perfectly ordinary physical system like any other and supposing ideal correlating interactions between the systems, which Everett explicitly did, the linear dynamics (4I) together with the dispositions of the ideal measuring device and ideal observer entail that the composite system $F+M+S$ evolve to the state

$$
\alpha\left|" \uparrow_{x} "\right\rangle_{F}\left|“ \uparrow_{x} "\right\rangle_{M}\left|\uparrow_{x}\right\rangle_{S}+\beta\left|“ \downarrow_{x} "\right\rangle_{F}\left|" \downarrow_{x} "\right\rangle_{M}\left|\downarrow_{x}\right\rangle_{S} .
$$

as the measuring device $M$ interacts with the object system $S$ and the friend $F$ interacts with the pointer on the $M$. So all that happens under the linear dynamics is that $M$ 's pointer and $F$ 's physical record of the measurement outcome become correlated to the $x$-spin of $S$ entangling the three systems. The standard interpretation of states (rule 3) tells us that this entangled state is not one where $F$ has a determinate measurement record. Indeed, since his record is entangled with both $M$ 's pointer position and $S$ 's $x$-spin, $F$ does not even have a proper quantummechanical state of his own. Hence one cannot explain the friend's experience by appealing to the state of his measurement record.

In contrast, if we suppose that there is something special about the friend that causes a collapse of the state of his object system on measurement, and consequently use the nonlinear collapse dynamics (4II) to characterize the interaction between $M$ and $F$, the composite system $F+M+S$ will either end up in state

$$
\left|" \uparrow_{x} "\right\rangle_{F}\left|" \uparrow_{x} "\right\rangle_{M}\left|\uparrow_{x}\right\rangle_{S}
$$

or in state

$$
\left|" \downarrow_{x} "\right\rangle_{F}\left|" \downarrow_{x} "\right\rangle_{M}\left|\downarrow_{x}\right\rangle_{S},
$$

with with probabilities $|\alpha|^{2}$ and $|\beta|^{2}$ respectively. Unlike the state resulting from the linear dynamics (2), each post-collapse state describes the friend $F$ as having a perfectly determinate measurement record on the standard interpretation of states. In the first, $F$ determinately records the result " $\uparrow$ " ", and in the second, he determinately records the result " $\downarrow_{x}$. "

The upshot is that dynamical laws (4I) and (4II) predict incompatible states when applied to the same interaction. If the standard theory told us precisely when to apply each dynamics, one might avoid the contradiction, but since measurement 
is an undefined primitive term in the theory, the standard formulation of quantum mechanics provides no guidance for when to use dynamical law (4I) and when to use dynamical law (4II). Everett, consequently, took the theory to be inconsistent and hence "untenable" (1955b, 59; 1956, 75). ${ }^{3}$

Everett's proposal for solving the measurement problem was simply to drop the collapse dynamics (4II) from the von Neumann-Dirac formulation and take there resultant theory to provide a complete and accurate description of all physical systems whatsoever. He called this theory pure wave mechanics. ${ }^{4}$

An immediate consequence of pure wave mechanics is that the composite system of the observer $F$, measuring device $M$, and system $S$ ends up in state (2) in the Wigner's Friend story above. Everett's goal then was to argue that the subjective appearances of an observer in such a post-measurement state would be the same as the experiences predicted by the collapse dynamics (4II) in the standard theory.

As he described his project in the long version of his $\mathrm{PhD}$ thesis, "we shall deduce the probabilistic assertions of [the collapse dynamics (4II)] as subjective appearances" to observers who are themselves treated as perfectly ordinary physical systems always subject to the linear dynamics (4I) "thus placing the theory in correspondence with experience." He noted that "[w]e are then led to the novel situation in which the formal theory is objectively continuous and causal, while subjectively discontinuous and probabilistic." And he took this to solve the quantum measurement problem because "while this point of view thus shall ultimately justify our use of the statistical assertions of the orthodox view, it enables us to do so in a logically consistent manner, allowing for the existence of other observers" and, hence, not leading to inconsistencies when one considers nested measurements of the sort described in the Wigner's Friend story $(1956,77-8)$.

\section{BRANCHES AND TYPICALITY}

While pure wave mechanics is manifestly logically consistent, it presents two new problems. The first is the determinate record problem. Addressing this problem involves explaining how measurement interactions produce determinate measurement records when the resultant state predicted by the linear dynamics (4I) is something like (2) above. The second is the probability problem. This involves explaining why measurement outcomes should be expected to exhibit the standard quantum

\footnotetext{
${ }^{3} \mathrm{~A}$ more charitable assessment would be to conclude that the theory is incomplete since it does not clearly indicate which law to use. It is only logically inconsistent if one insists, like Everett, that observers and measuring devices are physical systems like any other. Of course, this is a plausibly enough assumption. Note that Wigner believed that it was obvious how to complete the standard theory and ensure its consistency: simply stipulate that a conscious observer collapses the state of the system he observes.

${ }^{4} \mathrm{He}$ also referred to it as the theory of the universal wave function and as the relative-state formulation of quantum mechanics.
} 
probabilities when the theory predicts no stochastic events. Everett appealed to what he called cross sections and branches of the total state to explain determinate measurement records and he appealed to a typicality measure over these branches to explain the standard quantum statistics.

While pure wave mechanics does not describe an observer as having any particular absolute record after a typical measurement interaction, Everett took the theory to describe a post-measurement observer as having a number of different relative records. As he put it, in a typical post-measurement state there are "cross sections of the total wave function" in which each term or element in the superposition describes the observer with a definite relative measurement record that is correlated with a corresponding definite relative state of the observer's object system, where what he calls a cross section is just a particular decomposition of the total state of the composite system in terms of a selected orthonormal basis. ${ }^{5}$

In the Wigner Friend story above, the expression (2) describes a cross section of the total state, and each term describes a branch where both the friend and the measuring device have determinate relative measurement records. The first term describes a branch where $F$ records the relative result " $\uparrow_{x}$ " and the second term describes a branch where $F$ records the relative result " $\downarrow_{x}$."

While Everett never referred to branches as worlds in his published work, he clearly took the existence of cross sections that characterize branches where an observer has determinate relative measurement records as explaining the observer's determinate experience. As he put the point in conversation with Abner Shimony some years after finishing his thesis, "Each individual branch looks like a perfectly respectable world where definite things have happened" (Barrett and Byrne 2012, 275-6). If one thinks of each branch in expression (2) as representing a quantum world, then $F$ gets a perfectly determinate, but different, measurement record in each world. While there are a number of issues one would need to address to clarify Everett's account of determinate measurement outcomes, we will set these aside here in order to consider his account of quantum statistics. ${ }^{6}$

Everett clearly and repeatedly insisted that there were no probabilities in pure wave mechanics. Indeed, he took this point to be significant enough that he originally entitled his $\mathrm{PhD}$ thesis "Quantum Mechanics without Probability" (Barrett and Byrne 2012, 72). Rather than claim that his theory predicted the same chance events as predicted by the collapse dynamics (4II), Everett argued that it would

\footnotetext{
${ }^{5}$ Everett discussed cross sections and branches in an early working paper he wrote for Wheeler (Everett 1955b, 66-9). His distinction between absolute and relative states was the focus of the relative-state formulation of pure wave mechanics that he developed in his $\mathrm{PhD}$ thesis (Everett 1957, 173-96). And, as discussed below, Everett appealed to the notion of experiential worlds some years later in conversation with Abner Shimony (Barrett and Byrne 2012, 275-6).

${ }^{6}$ See Barrett (2011a, 2011b, and 2015) for further details regarding how Everett understood determinate measurement outcomes.
} 
appear to a typical observer that there were such chance events. More specifically, he argued that if an observer like $F$ were to perform a series of measurements, the sequence of the observer's relative measurement records in a typical branch, in the measure of typicality provided by the norm-squared of the coefficient associated with each branch in the determinate-record expansion of the total state, would appear to a particular relative observer to be randomly distributed with the standard quantum probabilities.

Everett took finding an appropriate notion of typicality for pure wave mechanics to be analogous to the problem of finding an appropriate typicality measure in classical statistical mechanics. As he put the problem:

In order to establish quantitative results, we must put some sort of measure (weighting) on the elements of a final superposition. This is necessary to be able to make assertions which hold for almost all of the observer states described by elements of a superposition. We wish to make quantitative statements about the relative frequencies of the different possible results of observation - which are recorded in the memory - for a typical observer state; but to accomplish this we must have a method for selecting a typical element from a superposition of orthogonal states (1956, 123-4; 1957, 190).

His strategy for finding such a measure was to impose a sequence of constraints until a particular measure was uniquely determined. Importantly, he did not argue that almost all branches by count will exhibit the standard quantum statistics; rather, he argued that almost all branches in a natural measure, given the structure of pure wave mechanics, will exhibit the standard quantum statistics.

While Everett clearly indicated that his typicality measure did not represent probabilities, he also stipulated that it satisfied the formal definition for a probability measure. He took his typicality measure $m$ to be a set function on subsets of the set of branches $B$ determined by a specified decomposition of the state. As a probability measure, it assigned a number between zero and one to each subset of $B$ such that $(1) m(B)=1$, and for subsets $Q$ and $R,(2) m(R)=1-m(\bar{R})$, and (3) if $R \cap Q=\emptyset$, then $m(R \cup Q)=m(R)+m(Q)$, and it was countably additive for any union of disjoint subsets of $B$.

His first constraint on the probability measure $m$ was that it be a positive function of the complex-valued coefficients $a_{i}$ associated with the branches of the superposition $\sum a_{i}\left|\chi_{i}\right\rangle$. This is natural enough inasmuch as, short of just counting the branches and considering relative proportions, pure wave mechanics does not provide much else that one might use to set the values for the typicality measure 
associated with branches structures. His second constraint was that $m$ be a function of the amplitudes of the coefficients alone. In particular, he required that $m\left(a_{i}\right)=m\left(\left|a_{i}\right|\right)$. The reason he gave was that the coefficients $a_{i}$ can only be empirically determined up to an arbitrary phase factor. Finally, he stipulated an additively condition. Inasmuch as any collection of branches $b_{i}$ of the total state can be considered to be a single branch $b^{*}$ on a different decomposition of the state, he stipulated that the measure assigned to $b^{*}$ be equal to the sum of the measures assigned to the branches $b_{i}$.

Synchronically, the additivity condition provides a natural nesting relation between the typicality measures assigned to branches on different cross sections or, equivalently, at different levels of description. Specifically, it ensures that the typicality measure assigns the same value to a relative property of a system regardless of whether that property is associate with one branch on one decomposition or $k$ branches on another. ${ }^{7}$

Diachronically, the additivity condition ensures a suitable conservation of typicality of branches under the linear dynamics. As Everett put the point:

$[\mathrm{W}] \mathrm{e}$ wish to make statements about 'trajectories' of observers. However, for us a trajectory is constantly branching (transforming from state to superposition) with each successive measurement. To have a requirement analogous to the 'conservation of probability' in the classical case, we demand that the measure assigned to a trajectory at one time shall equal the sum of the measures of its separate branches at a later time. This is precisely the additivity requirement which we imposed and which leads uniquely to the choice of square-amplitude measure. Our procedure is therefore quite as justified as that of classical statistical mechanics (1956, $126 ; 1957,192)$.

Everett took these three constraints to determine uniquely the typicality measure $m$ as the norm-squared-amplitude measure on branches. Putting the pieces together, then, he thought of measurement as a process that forms increasing more complex superpositions of relative measurement outcomes. In the determinaterecord basis, this involves the constant branching of an observer's trajectory where he gets different sequences of relative results in each branch. And the measure associated with each branch is equal to the norm-squared of the coefficient on the term describing that branch in the expression of the composite system's total state. Figure 1 below represents the branching structure one gets for a sequence of three measurements of three systems each initially in state (5).

${ }^{7}$ Note that since there is no canonical decomposition of the total state, one needs a different typicality measure for each possible cross-section. 


\section{THE TYPICALITY OF BRANCHES}

In both the long and short versions of his thesis, Everett explicitly stipulated that the measure $m$ does not represent probabilities. ${ }^{8}$ Indeed, inasmuch as he took all branches to be equally actual, he simply took the probability of each branch to be one. ${ }^{9}$ Pure wave mechanics is fully deterministic and postulates no chance events, and, at least for his part, Everett did not take $m$ to represent the subjective credences of an agent either. Rather, he simply took it to be a necessary prerequisite for talking sensibly about the properties of typical branches.

Everett's main result was that almost all branches in measure will exhibit the standard quantum statistics in the limit as the number of measurements one has performed gets large. This was the key point of his thesis and the fact that he thought was most significant in understanding pure wave mechanics. ${ }^{10}$

While Everett himself provided only a sketch of the argument, those limiting properties of pure wave mechanics that he found most salient can be proven. ${ }^{11}$ In the case of the $x$-spin example above, consider a measuring device $M$ that is ready to make an record an infinite series of measurements on each of an infinite series of systems $S_{k}$ in state

$$
\alpha\left|\uparrow_{x}\right\rangle_{S_{k}}+\beta\left|\downarrow_{x}\right\rangle_{S_{k}} .
$$

Suppose that $M$ interacts with each system in turn and perfectly correlates its $k$ th memory register with the value of system $S_{k}$ by the linear dynamics (4I). This will produce an increasingly complicated entangled superposition of different sequences of measurement outcomes. After one measurement, the state of $M$ and $S_{1}$ will be

$$
\alpha|" \uparrow "\rangle_{M}|\uparrow\rangle_{S_{1}}+\beta|" \downarrow "\rangle_{M}|\downarrow\rangle_{S_{2}} .
$$

After two measurements, the state of $M, S_{1}$, and $S_{2}$ will be

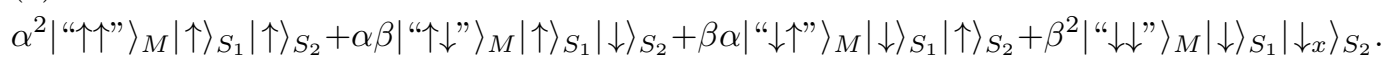

And so on. In the determinant-record basis, there will be $2^{n}$ terms after $n$ measurements, one term corresponding to each possible sequence of possible measurement

\footnotetext{
${ }^{8}$ See, for example, Everett $(1956,127)$ and $(1957,193)$.

${ }^{9}$ Everett took all branches to be equally actual because, as he explained in the long version of his thesis, he believed that it was always in principle possible to observe interference effects between elements of a superposition $(1956,149)$. Regarding the reality of all branches, see also the note Everett added in proof to the short version of his thesis (1957, 189). Albert (1986) used Everett's commitment to the ever-present possibility of detecting interference between branches to argue that Everettian worlds cannot be considered to be causally isolated. For a closely-related argument see Albert and Barrett (1995).

${ }^{10}$ See the transcript of Everett's discussion of this point at the Xavier Conference (Barrett and Byrne 2012, 274-5).

${ }^{11}$ See, for example, Hartle (1968), DeWitt (1971), Farhi, Goldstone, and Gutmann (1989). See Barrett (1999, 95-107) for proofs of the two results described below.
} 
outcomes. Figure 1 illustrates the first three steps of the branching process and the amplitudes associated with each branch.

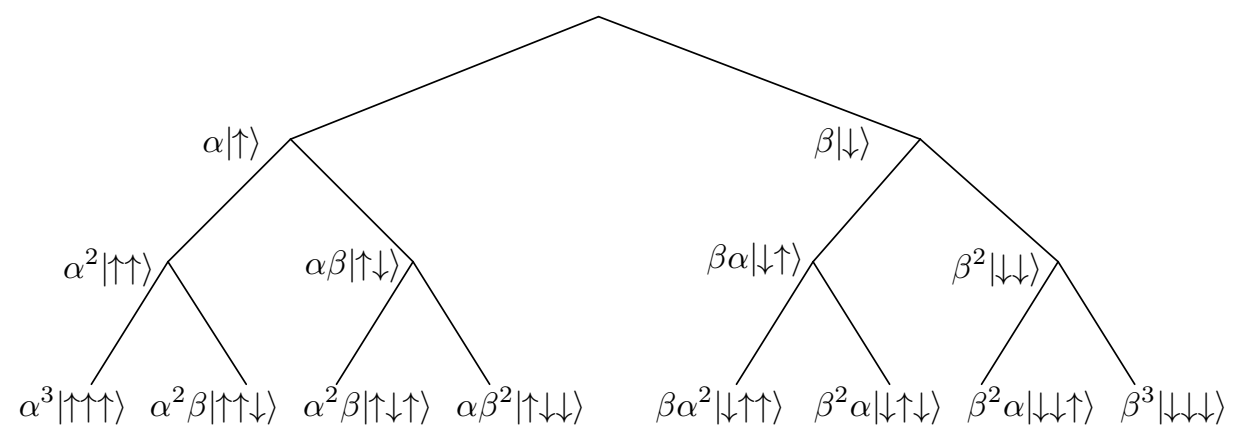

FiguRE 1. Branching on repeated measurements

One can show that for any $\delta>0$ and $\epsilon>0$, there exists a $k$ such that after $k$ measurements the sum of the norm-squared of the amplitude associated with each branch where the distribution of spin-up results is within $\epsilon$ of $|\alpha|^{2}$ and the distribution of spin-down results is within $\epsilon$ of $|\beta|^{2}$ is within $\delta$ of one. One can also show that the sum of the norm-squared of the amplitude associated with each branch where the sequence of relative records is algorithmically incompressible, or satisfies any other of the standard criteria for being random, goes to one as the number of measurements gets large. The upshot is that in the limit as the number of measurements gets large, almost all branches in norm-squared coefficient measure will describe sequences of measurement records that are randomly distributed with the standard quantum statistics.

Note that it is typically not the case that most branches by count will exhibit the standard quantum statistics. ${ }^{12}$ This fact led Bryce DeWitt (1971) and Neill Graham (1973) to argue that Everett's typicality measure was unmotivated. As Graham put it, "it is extremely difficult to see what significance such a measure can have when its implications are completely contradicted by a simple count the worlds involved." 13

What DeWitt and Graham wanted was a theory where the simple proportion of worlds that exhibit the standard quantum statistics goes to one in the limit as the number of measurements gets large. Only then did they believe that one would have an adequate account of the appearance of the standard quantum statistics. As a result of this commitment, DeWitt and Graham (1973) described an alternative

${ }^{12}$ Here most branches by count will exhibit the standard quantum statistics if $|\alpha|^{2}=|\beta|^{2}=1 / 2$ but not if $|\alpha|^{2} \neq|\beta|^{2}$.

${ }^{13}$ See Barrett and Byrne $(2012,366)$ for a reproduction of the relevant section of Graham (1973). 
splitting-worlds formulation of quantum mechanics where the number of worlds that exhibit a particular outcome after a measurement interaction is proportional to the square of the coefficient on the term describing that outcome. This ad hoc splitting procedure ensures that almost all worlds by simple count will exhibit the standard quantum statistics. ${ }^{14}$

For his part, Everett held that DeWitt and Graham were deeply confused about how statistical explanation worked in pure wave mechanics. In response to the passage quoted from Graham above, Everett wrote "bullshit" in the margin of his personal copy of the paper. ${ }^{15}$ Inasmuch as he simply wanted to show that a typical branch will exhibit the standard quantum statistics, Everett was certainly right that he could use whatever typicality measure he wanted. Further, as he took pains to argue, the typicality measure he used was natural given the structure of pure wave mechanics.

That DeWitt and Graham believed that making something true of most branches by count was the only way to explain why an observer should expect his results to exhibit the standard quantum statistics tells us something about their background commitments. To begin, they clearly took their own talk of worlds inhabited by copies of the initial observer seriously. Second, they seem to have wanted standard probabilistic predictions from their theory. And finally, there is also good reason to believe that they were guided in this by familiar, but confused, intuition. This intuition is sometimes called a principle of indifference. Such a principle stipulates that if there are $n$ possible outcomes of an event, then, in the absence of other information, one must assign probability $1 / n$ to each possibility.

If one believed in splitting worlds and believed that quantum probabilities concerned the relative likelihood of finding oneself in a future world with specified properties, and if one could make clear sense of both of these commitments, then if one held a principle of indifference as a stipulation regarding the rational assignment probabilities over alternative future worlds, then one might conclude that the self-location probability associate with each branch should be equal. ${ }^{16}$ In that case, one might believe that the probability of finding oneself associated with a branch that exhibits the standard quantum statistics is high if and only if most branches by count exhibit the standard quantum statistics.

There are, however, a number of problems with this line of argument. First, a principle of indifference always depends on a prior choice of a partition. Since pure

\footnotetext{
${ }^{14} \mathrm{On}$ the assumption that one restricts the theory to measurement interactions that produce at most a finite number of branches.

${ }^{15}$ Everett's copy of the paper was in his copy of the DeWitt and Graham (1973) anthology on the many-world interpretation. See Barrett and Byrne 2012, 364-6 for photographs of Everett's notes.

${ }^{16}$ See Saunders, Barrett, Kent, and Wallace (eds) (2010) for a review of what one might mean by worlds and self-location probabilities in the context of a many-worlds reformulation of Everett.
} 
wave mechanics specifies no single, canonical decomposition of the full state into branches, one does not even know what set of branches one should use in assigning each branch probability $1 / n .{ }^{17}$ Second, principles of indifference are typically not considered to be constraints on rationality. A good Bayesian might, for example, assign any set of coherent, non-dogmatic priors to $n$ hypotheses without fear of finding herself committed to a Dutch book. Consequently, if one wants to appeal to a principle of indifference to get probabilities in pure wave mechanics, then one cannot plausibly appeal to it as a prior, accepted rule of rationality; rather, honesty would require that one add the principle to the theory explicitly. Finally, but most significantly, it would have been inappropriate for Everett to appeal to a principle of indifference in order to introduce probabilities into his theory inasmuch as he was concerned with the typicality of branches not their probability.

We will briefly consider the relationship between typicality and probability in the next section. Here the suggestion is just that since Everett was interested in the typicality and not probability, he was free to use any notion of typicality at hand and the line of argument pursued by DeWitt and Graham has little purchase. Given the structure of pure wave mechanics, the norm-squared typicality measure Everett chose was natural, but, as he knew, it was not the only measure one might consider.

\section{FROM TYPICALITY TO PROBABILITY}

Showing that the standard quantum statistics can be found in a typical branch does not entail anything about probabilities or expectations. The issue is not one's choice of typicality measure, as DeWitt and Graham supposed, but rather the inferential move from what is true in a typical branch to what one should expect. For Everett, it was enough to show that almost all determinate-record branches in the norm-squared coefficient measure will exhibit the quantum statistics for pure wave mechanics to be considered empirically faithful. But if one wanted a theory that predicted the same probabilities as the standard collapse formulation of quantum mechanics, one would need to add an assumption to the theory that tied branch typically to chance or expectation.

One way to see the point is to note that while the greatest measure of determinaterecord branches of the total state will exhibit the standard quantum statistics after

\footnotetext{
${ }^{17}$ Note that this is also true on decoherence formulations of the many-worlds interpretation like David Wallace's (2014) inasmuch as there is again no simple matter of fact concerning how many worlds there are. Note also that if one allows for the possibility of a countably infinite number of worlds and assumes that probabilities are countably additive, then, again one cannot consistently apply the principle of indifference. See Saunders, Barrett, Kent, and Wallace (eds) (2010) for a review of a number of approaches to making sense of probability in various Everettian formulations of quantum mechanics.
} 
a series of measurements, many branches will not. In the repeated $x$-spin measurements above, for example, there will always be a branch where the observer got $x$-spin up as the result of every measurement. Such relative records would not exhibit the standard quantum statistics. One wants to say that it is unlikely that one's experiences would be represented by such a branch, but the probability of the branch being fully realized on given the linear dynamics (4I) is simply one.

In some respects the task here has an analogue in classical statistical mechanics. Just because one has a typicality measure such that entropy-increasing trajectories for a given initial macro-state are typical in the measure does not by itself entail that such trajectories are probable. Rather, to get probabilistic predictions from the theory, one must add an assumption that allows one to infer probabilities from the typicality measure, something that says that given appropriate conditions one should expect a system to be typical in the specified sense. ${ }^{18}$

Similarly, the most direct way to get from pure wave mechanics to a theory that allows one to infer that it is probable that one's determinate relative records will exhibit the standard quantum probabilities is to suppose that one's actual relative records should be expected to be typical in the sense Everett specified. Along these lines, one might propose adding to the theory something like the assumption that one's own future self-located relative-record state is randomly selected in the normsquared coefficient measure. That said, since all branches are equally actual in pure wave mechanics, there is nothing in the description of the world provided by pure wave mechanics that would mark any branch as the one that is randomly selected at the exclusion of the other options.

This is in contrast with a hidden-variable theory like Bohmian mechanics (1952) where a parameter, in this case the total particle configuration, selects one branch as the effective wave function at a time. Everett, however, explicitly rejected Bohmian mechanics on "grounds of simplicity" precisely because it adds a new parameter to the theory and hence is more complicated than pure wave mechanics, which, of course, he takes to already be empirically faithful $(1956,154)$.

The upshot is that while pure wave mechanics does not predict that any particular outcome is realized with the standard quantum probabilities at the expense of alternative possibilities, one can get probabilistic predictions regarding one's experience if one explicitly adds something to the theory that ties the typicality measure to one's expectations of future measurement outcomes. But if one does so, such probabilities will not characterize the likelihood of events occurring that

\footnotetext{
${ }^{18}$ In the context of classical statistical mechanics, this is known as the statistical assumption. See Albert (2001) for a discussion of the difficulties one encounters in providing an adequate specification of the statistical assumption.
} 
are described by pure wave mechanics. Again, every event described by the theory simply occurs.

\section{Conclusion}

While pure wave mechanics is a deterministic theory that explicitly involves no assumptions regarding probability, one can show that if an observer performs a series of ideal measurements, in the limit as the number of measurements gets large almost all determinate-record branches will exhibit the standard quantum statistics. For Everett, this was sufficient to establish the empirical faithfulness of the theory. But this does not mean that the theory allows one to infer the standard probabilistic predictions of quantum mechanics for the outcomes of future measurements.

The most direct way to get probabilities would be to add an assumption to pure wave mechanics that explicitly connects the typicality measure Everett specified to one's expectations. One might, for example, assume that one's experience should be expected to correspond with precisely one future determinate-record branch randomly selected in the norm-squared coefficient measure. But if one further requires that the theory provide the resources for saying which branch was randomly selected, one would also need to add a parameter that specifies the actual branch. Hidden-variable theories like Bohmian mechanics do precisely this, but Everett explicitly took such theories to lack the simplicity and naturalness of pure wave mechanics precisely because they added something to pure wave mechanics that it did not need.

One might, of course, settle for Everett's solution. On this view, one simply accepts the explanation of the statistical predictions of quantum mechanics as branch typical rather than probable. 
Bibliography

Albert, David, Z (2001) Time and Chance. Cambridge, MA: Harvard University Press.

Albert, David, Z (1986) "How to Take a Photograph of Another Everett World" Annals of the New York Academy of Sciences: New Techniques and Ideas in Quantum Measurement Theory 480: 498-502.

Albert, David Z and Jeffrey A. Barrett (1995) "On What It Takes to Be a World," Topoi, 14(1): 35-37.

Barrett, Jeffrey A. (2011a) On the Faithful Interpretation of Pure Wave Mechanics British Journal for the Philosophy of Science 62 (4): 693-709.

Barrett, Jeffrey A. (2011b) Everett's Pure Wave Mechanics and the Notion of Worlds European Journal for Philosophy of Science 1 (2):277-302.

Barrett, J. A. (2015) Pure Wave Mechanics and the Very Idea of Empirical Adequacy, forthcoming in Synthese.

Barrett, Jeffrey A. (1999) The Quantum Mechanics of Minds and Worlds, Oxford: Oxford University Press.

Barrett, Jeffrey A. and Peter Byrne (eds) (2012) The Everett Interpretation of Quantum Mechanics: Collected Works 1955-1980 with Commentary, Princeton: Princeton University Press.

Bohm, David (1952) "A Suggested Interpretation of Quantum Theory in Terms of 'Hidden Variables'," Parts I and II, Physical Review 85, 166-179, 180-193.

DeWitt, Bryce S. (1971) "The Many-Universes Interpretation of Quantum Mechanics" in B. D. 'Espagnat (ed.) Foundations of Quantum Mechanics. New York: Academic Press. Reprinted in DeWitt and Graham (1973) pp. 167-218.

DeWitt, Bryce S. and Neill Graham (eds.) (1973) The Many-Worlds Interpretation of Quantum Mechanics. Princeton: Princeton University Press. 
Dirac, P. A. M. (1930) The Principles of Quantum Mechanics. Oxford: Oxford University Press.

Everett, Hugh III (1955a) "Probability in Wave Mechanics" Undated typescript addressed to John Wheeler with Wheeler's marginal notes. In Barrett and Byrne (eds) $(2012,64-70)$.

Everett, Hugh III (1955b) "Objective vs Subjective Probability" Undated typescript addressed to John Wheeler with Wheeler's marginal notes. In Barrett and Byrne (eds) (2012, 57-60).

Everett, Hugh III (1956) "The Theory of the Universal Wave Function." In Barrett and Byrne (eds) (2012, 72-172).

Everett, Hugh III (1957) " 'Relative State' Formulation of Quantum Mechanics," Reviews of Modern Physics, 29: 454-462. In Barrett and Byrne (eds) (2012, 17396).

Farhi, E., J. Goldstone, S. Gutmann (1989) "How Probability Arises in Quantum Mechanics," Annals of Physics 192(2) 368-382.

Graham, N. (1973) "The Measurement of Relative Frequency," in DeWitt and Graham (1973).

Hartle, J. B.: (1968) "Quantum Mechanics of Individual Systems," American Journal of Physics, 36(8) 704-12.

Saunders, Simon; Jonathan Barrett; Adrian Kent; David Wallace (eds) (2010) Many Worlds?: Everett, Quantum Theory, and Reality, Oxford: Oxford University Press.

von Neumann, J. (1955) Mathematical Foundations of Quantum Mechanics. Princeton: Princeton University Press. Translated by R. Beyer from Mathematische Grundlagen der Quantenmechanik. Berlin: Springer (1932).

Wallace, David (2012) The Emergent Multiverse: Quantum Theory according to the Everett Interpretation, Oxford: Oxford University Press.

Wigner, Eugene (1961) "Remarks on the Mind-Body Problem", in I. J. Good (ed.), The Scientist Speculates, New York: Basic Books, pp. 284-302. 